\title{
Image Matching Algorithm for Remote Sensing based on FAST-9 and SURF
}

\author{
Hao Wu ${ }^{\text {a }}$, Wei Yang b, Jun Liu b \\ ${ }^{a}$ School of Computer \& communication Engineering, University of Science \& Technology Beijing, \\ Beijing 100081, China \\ ${ }^{\mathrm{b}}$ No. 145, Erqi Road, Wuhan, Hubei, 430012, China
}

Keywords: Remote Sensing; Image Matching; FAST-9; SURF.

\begin{abstract}
A novel remote sensing image matching algorithm based on FAST-9 and SURF is proposed. Firstly, the FAST-9 is employed to detect feature points for remote sensing images, and the ID3 algorithm and non maximum method are used to determine the correct corners. And then, main orientation is assigned by the circular region around the interest point. The SURF descriptor is constructed by the square region aligned to the selected orientation. Finally, the matching pairs are calculated by the nearest neighbor distance matching method. Experimental results show that the proposed matching algorithm is effective for remote sensing images, which has excellent invariance.
\end{abstract}

\section{Introduction}

Remote sensing image has been more and more widely used in resources survey, disaster monitoring and military reconnaissance and other fields. Image matching is one of the key technologies in the field of remote sensing image processing, which plays a vital role in the multi-source remote sensing image registration, image stitching. In practical applications, the presence of rotation, viewpoint, scaling, translation, conversion of light, noise and other differences between most of the images to be matched, brings great difficulty to image matching. The reference [1] proposed a rotation invariant LBP algorithm for global match. Extract the main direction of the image, construct LBP neighborhood and get its value according to the main direction, use the histogram texture information to generate LBP descriptors. The proposed algorithm can improve accuracy of $10 \%$ compared with the conventional matching algorithm. Wang Wei et al [2] presented an image description and recognition algorithm based on the characteristics of LBP pyramid. Face image pyramid is constructed by multi-scale analysis, using layers of images to extract LBP operator characteristic spectrum of LBP. The feature spectrum of block statistics is calculated, and histograms are cascaded sequence for classification. For remote sensing image matching problem, a novel image matching algorithm for remote sensing based on the FAST-9 and SURF is proposed. Feature points are detected by the FAST-9, and then described via the use of SURF, to achieve much accurate matching results ultimately.

\section{FAST-9 detector}

Rosten and Drummond present a simple and fast FAST (Features from Accelerated Segment Test) corner detection algorithm [3] based on SUSAN (Smallest Univalue Segment Assimilating Nuclues). Compared with the other corner detectors, the FAST detector can improve the detection rate by the sub-guarantee repeatability and noise immunity to a certain extent. Then Rosten introduced a machine learning and ID3 decision tree [4] to improve the FAST detector, which further improves the speed of the algorithm. Specific steps of FAST-9 are shown below.

Step1. Select any point on the image of 16 pixels as templates, shown in Figure 2, when the center point of the circular area of the template case, where $n=9, r=3$. $P$ is in the center of the template with 16 pixels, with respect to each pixel $p$ is concerned can be divided into three states: darker, similar, and lighter than $p$. Then for each pixel in the 16 pixel template, $x \in\{1,2, \ldots, 16\}$, which is referred to as the relative position of $p \rightarrow x$, and the three states can be written as follows: 


$$
S_{p \rightarrow x}=\left\{\begin{array}{cc}
d, & I_{p \rightarrow x} \leq I_{p}-t \\
s, & I_{p}-t<I_{p \rightarrow x}<I_{p}+t \\
b, & I_{p}+t \leq I_{p \rightarrow x}
\end{array}\right.
$$

Where, $t$ is a threshold of gray level difference, as the case may be adaptively set according to the detected image; $d$ indicates the current point darker than the center point; $s$ indicates the current point has similar gray; and $b$ indicates the current lighter.

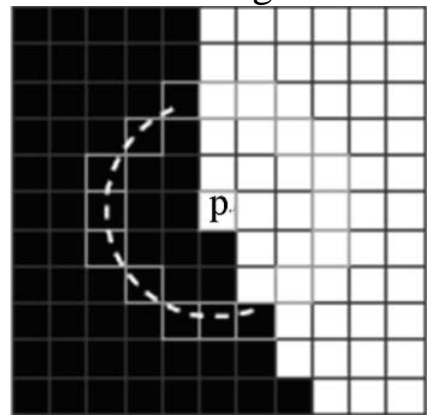

Fig.1 FAST-9 detector

For each pixel is calculated on the image according to the formula (1), all pixels set $P$ of the entire image can be divided into three subsets, wherein all pixels brighter than the center set of pixels is defined as:

$$
P_{b}=\left\{p \in P: S_{p \rightarrow x}=b\right\}(2)
$$

$P_{d}$ and $P_{s}$ can be defined as the same. Set $K_{p}$ to be a boolean variable, when the detector $p$ is the corner its value is true, otherwise false.

Step2. Use ID3 algorithm to determine whether the candidate pixel is corner, based on the entropy of the point $K_{i}$ is positive or negative. An arbitrary set of pixels of the total entropy is defined as:

$$
H(Q)=(c+\bar{c}) \log _{2}(c+\bar{c})-c \log _{2} c-\bar{c} \log _{2} \bar{c}(3)
$$

where $c=\mid\left\{i \in Q: K_{i}=\right.$ true $\}\left(\right.$ Num $\left._{\text {corner }}\right), \bar{c}=\mid\left\{i \in Q: K_{i}=\right.$ false $\} \mid\left(\right.$ Num $\left._{\text {noncorner }}\right)$. Then make the information gain the greatest attribute as a classification attribute $\mathrm{x}$ is calculated according to the following formula, namely:

$$
H_{g}=H(P)-H\left(P_{d}\right)-H\left(P_{s}\right)-H\left(P_{b}\right)(4)
$$

Thus the above three sets can be divided into three subsets, for example, $x_{b}$ will be divided into $P_{b, d}, P_{b, s}$, and $P_{b, b}$. The algorithm is iterated in three sets, and iterative process continues until a subset of entropy is zero so far., as the subset of values $K_{p}$ are the same, so the collection of pixels can be classified as all corners or non-corners.

Step3. The optimum corners are selected from repeated ones via non-maximum suppression, to form the final set. Intuitively considered poor corner with gray pixels around the neighborhood and the largest, and therefore we firstly establish the evaluation function:

$$
E=\sum_{x \in J}|I(x)-I(p)|(5)
$$

where, $J$ is a collection of eight pixels around the center point $p$ of the neighborhood and all corners in $3 \times 3$ area are calculated according to equation (5), and then keep the value of the maximum point as the final corner, and delete all the other corners of the neighborhood.

\section{SURF based matching algorithm for remote sensing images}

\subsection{SURF descriptors}

Lowe proposed scale invariant feature (Scale Invariant Feature Transform, SIFT) algorithm. SIFT algorithm can effectively extract local invariant features of the image, but because of its large amount of calculation and the descriptors of high dimensionality, which greatly influenced the matching 
speed. To solve this problem, Bay et al in 2006 proposed the SURF (Speeded-Up Robust Feature) algorithm [5], which are close to or exceed the performance of the various aspects of SIFT.

In this paper, SURF descriptor is employed to describe FAST-9 features. To make the feature point descriptors rotation invariant, each main direction is allocated to feature points. Allocation process can be divided into the following steps:

Step1. To set a feature point as the center, in the neighborhood of the circle with the radius 6c (c corresponding to the characteristic point scale), the image is processed with Haar wavelet templates of scale 4c. And get the Haar wavelet response in both $\mathrm{x}$ and $\mathrm{y}$ directions. Then use Gaussian function with $\sigma=2 c$ to weight these responses. Haar wavelet template is shown in Figure 4.

Step2. Feature points as the center of a circle with a sector angle $\pi / 3$ of the sliding window, in step 0.2 radian around a week, which is calculated at each angle including all the feature point. These constitute a direction vector and the response of which the longest is the main direction of the vector corresponding to the feature point.

When generating descriptors, a side length with 20c of the rectangular region is constructed. Along the main direction, the rectangular area is divided into $4 \times 4$ sub-regions, which has $5 c \times 5 c$ pixel. The Haar wavelet template with size 2c is used to calculate the response of the sub block. Then, 25 samples are carried out to get the main direction $d y$ and perpendicular to the main direction $d x$. And then, calculate the Gaussian-weighted basis at the center of feature points, where, $\sigma=3.3 c$. Finally, in response to the value of each sub-area statistics we get a 4-dimensional vector $V_{s}=\left[\sum d x, \sum|d x|, \sum d y, \sum|d y|\right]$, as the 64-dimensional feature descriptors.

The Euclidean distance of descriptors is employed to be the determining similarity measure of the feature points in images. Select one feature point in matching image, and find the nearest and less points in the candidate image by Euclidean distance. Suppose that the distances are $D_{\text {FirMin }}$ and $D_{\text {SecMin }}$, if their ratio is not greater than a certain threshold value $T$, then consider that the feature points to be matched with the minimum distance matches the feature point.

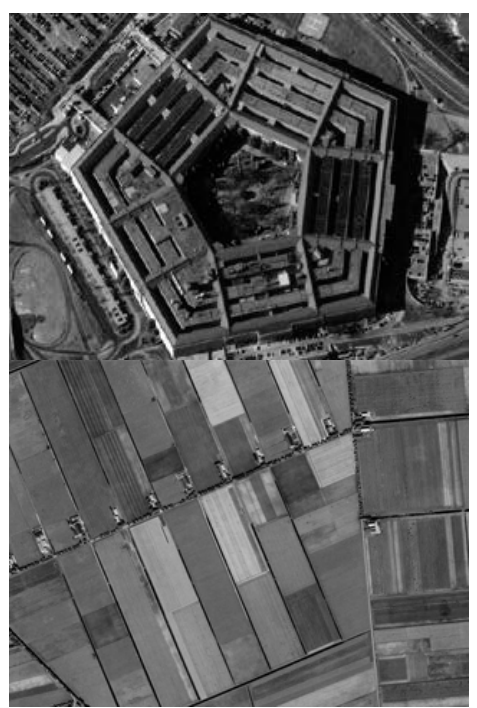

(a) sensing artwork

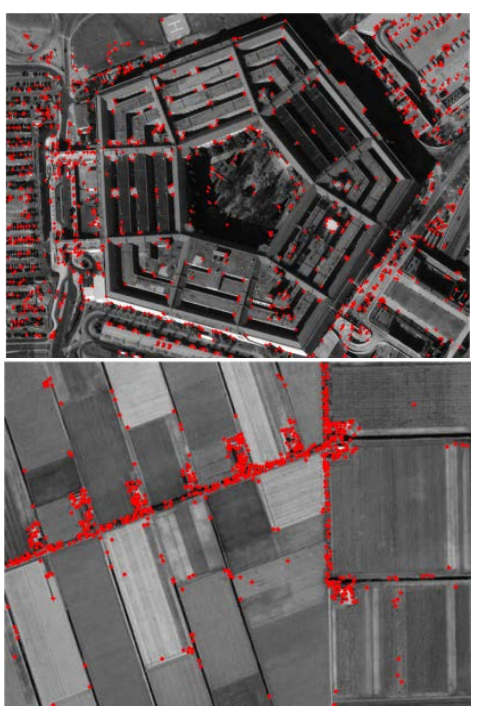

(b) FAST-9 test results (c) rotated 45 degrees test results

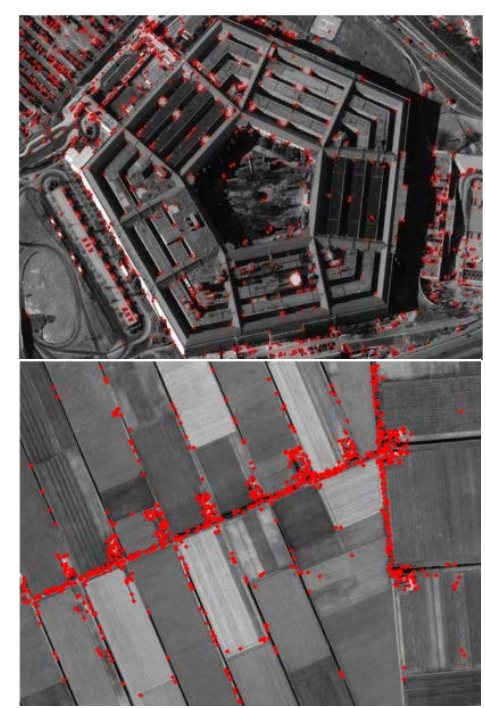

Figure 2 FAST-9 remote sensing image corner detection results (from top to bottom, respectively

Pentagon image, farmland image)

Tab.1 FAST-9 experimental results

\begin{tabular}{|c|c|c|c|}
\hline Remote sensing image & corner points & detection time & Image Resolution \\
\hline Pentagon & 982 & $1.645 \mathrm{~s}$ & $640 \times 480$ \\
\hline Pentagon rotation $45^{\circ}$ & 1168 & $1.745 \mathrm{~s}$ & $640 \times 480$ \\
\hline Farmland & 580 & $1.050 \mathrm{~s}$ & $480 \times 360$ \\
\hline Farmland rotation $45^{\circ}$ & 724 & $1.041 \mathrm{~s}$ & $480 \times 360$ \\
\hline
\end{tabular}




\subsection{Experimental results and analysis}

To verify the effectiveness of the matching algorithm, the experiments are processing under CPU Core2 Duo 2.80GHz, RAM 1GB, Windows XP + SP3, and VC ++6.0 . FAST-9 test results are shown in Figure 2 and Table 1. As can be seen, the use of FAST-9 detectors can detect a large number of sub-stable feature points.

The proposed matching algorithm has perspective performance, such as rotation and transformation of light, as shown in Figure 3. The algorithm can get change invariance excellencely.

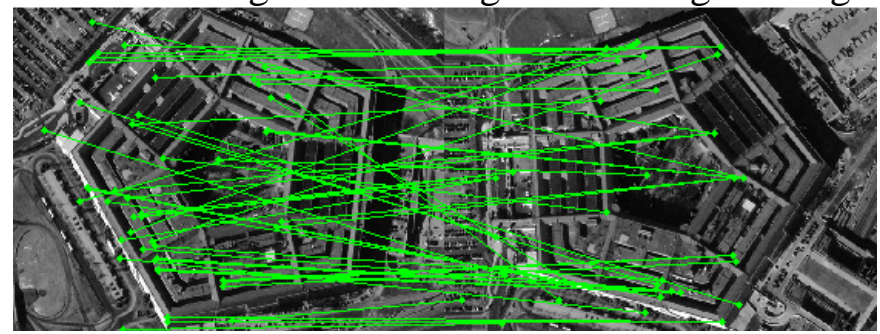

(a) image matching results of Pentagon

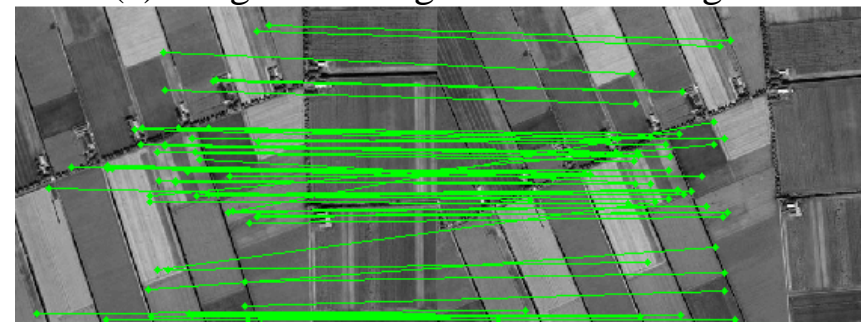

(b) image matching results of Farmland

Fig. 3 image matching results for remote sensing images

Remote sensing image matching results are shown in Table 2, from which we can see the validity of this method.

Table 2 matching results for remote sensing images

\begin{tabular}{|c|c|c|c|}
\hline Remote sensing images & matching points & $\begin{array}{c}\text { generating time of } \\
\text { descriptor }\end{array}$ & matching time \\
\hline Pentagon & 68 & $3.054 \mathrm{~s}$ & $0.016 \mathrm{~s}$ \\
\hline Farmland & 56 & $6.258 \mathrm{~s}$ & $0.875 \mathrm{~s}$ \\
\hline
\end{tabular}

\section{Conclusion}

For remote sensing image matching problem, a remote sensing image matching algorithm based on the FAST-9 and SURF. Firstly, FAST-9 remote sensing image feature detection, and then use the template Haar wavelet image processing by constructing SURF descriptor, using Euclidean distance as a measure of similarity judgment image feature points, and ultimately effective remote sensing images match. Experimental results demonstrate the effectiveness of this method. The next step is to further improve the research focused mainly on the real-time algorithm.

\section{References}

[1] Zhenhua Guo, Lei Zhang, David Zhang. Rotation invariant texture classification using LBP variance (LBPV) with global matching[J]. Pattern Recognition, 2010, 43: 706-719.

[2] Wang Wei, Huang Feifei, LI Jian, FENG Hai-liang pyramid using LBP face description and recognition [J] Computer-Aided Design and Computer Graphics, 2009, 21 (1): 94-102.

[3] Rosten E, Drummond T, Fusing points and lines for high performance tracking[J]. Proceedings of the International Conference on Computer Vision, 2005, 1508-1511.

[4] Rosten E, Drummond T, Machine learning for high-speed corner detection[J]. Proceedings of the European Conference on Computer Vision, 2006, 430-443.

[5] Herbert Bay, Tinne Tuytelaars, Luc Van Gool. SURF: Speeded Up Robust Features[C] 9th European Conference on Computer Vision, Graz, Austria, ECCV, 2006: 404-417. 